\title{
A Novel Global Self-Timing Methodology for BSFQ Circuits
}

\author{
Chen Kong Teh and Yoichi Okabe, Member, IEEE
}

\begin{abstract}
Recently we have proposed Boolean single-fluxquantum (BSFQ) circuits, which like CMOS circuits directly support Boolean primitives, and do not require local synchronization for their elementary cells as well as for their combinational cells. However, only the cell-level timing description of the BSFQ circuits was considered, which did not specify their global timing strategy in a system-level design. In this paper, we present a novel global self-timing methodology, dual encoding hierarchical pipelining (DEHP), for the locally asynchronous BSFQ circuits. In circuit implementation, a nonvolatile memory cell named ND-DFF and a volatile memory cell named D-DFF have been designed.
\end{abstract}

Index Terms-Asynchronous design, Boolean primitive, BSFQ, pipelining, self-timing, SFQ circuits.

\section{INTRODUCTION}

$\mathbf{R}$ ECENTLY we have proposed Boolean single-fluxquantum (BSFQ) circuits [1], [2], which like CMOS circuits directly support Boolean primitives, and do not require local synchronization for their elementary cells as well as their combinational cells. Moreover, BSFQ circuits are also characterized by their simple design, low latency, high modularity, and high flexibility in terms of easily interfacing with other types of SFQ circuits. However, despite their locally asynchronous feature, BSFQ circuits, similar to CMOS circuits, necessitate a global timing signal for a system with sequential circuits. In our previous work, we only considered BSFQ circuits as independent circuits themselves, and did not specify their global timing strategy in a system-level design. Therefore, in order to provide BSFQ circuits with a globally asynchronous feature, we have developed a novel global self-timing methodology, named dual encoding hierarchical pipelining (DEHP).

In the context of global timing strategy, BSFQ logic can utilize both synchronous and asynchronous timing scheme used by CMOS logic. However, synchronous timing scheme requires severe attention for clock skew problem at multi-gigahertz operating frequencies, and restricts a system to a worse case performance. On the other hand, despite allowing average-case performance, CMOS asynchronous timing scheme requires rather bulky hardware and complicated design for control logic subsystem. These facts motivate us to develop an alternative globally asynchronous timing scheme, which enables BSFQ system

Manuscript received August 6, 2002. This work was supported in part by a grant from the Superconductivity Research Laboratory, Japan.

C. K. Teh is with the Electronic Engineering Department, University of Tokyo, Tokyo 113-8656, Japan (e-mail: teh@okabe.rcast.u-tokyo.ac.jp).

Y. Okabe is with the University of Tokyo, Tokyo 113-8656, Japan (e-mail: okabe@okabe.rcast.u-tokyo.ac.jp).

Digital Object Identifier 10.1109/TASC.2003.813931 (a)

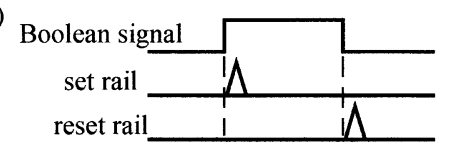

(b)

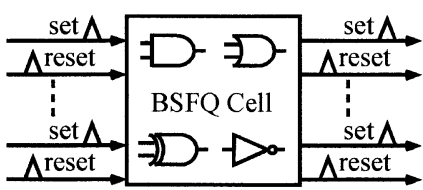

c)

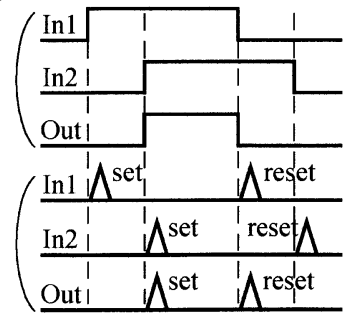

Fig. 1. (a) BSFQ local encoding scheme. (b) Basic diagram of a BSFQ cell. (c) Usage of BSFQ local encoding scheme is shown by using dynamics of an AND gate.

to have high throughput and low latency, without too much complexity overhead.

The first idea of DEHP methodology is to use two suitable encoding schemes, which can provide required timing information for combinational circuits and sequential circuits, respectively. The second idea is to use self-timed hierarchical pipelines, comprised of micro-pipelines and meta-pipelines, for building an arbitrary BSFQ system.

\section{Cell-Level Timing Scheme}

SFQ circuits inherit different operating fashion compared to that of CMOS circuits, where the former uses voltage pulse for data transmission, while the latter uses voltage level. In SFQ circuits, since the arrival of a SFQ pulse is at a point in time, synchronizing signals are important for establishing timing windows for data signals. On the other hand, in CMOS circuits, the time interval of a level signal behaves as a timing window for itself, and an overall timing window is obtained by overlapping timing windows produced by all input signals.

By this consideration, BSFQ logic adopts level-processing methodology of CMOS logic, on top of its pulse-based operating mode. In BSFQ logic, a Boolean level signal is represented by a "set" SFQ pulse at the rising edge of the signal, and a "reset" SFQ pulse at the falling edge of the signal [Fig. 1(a)]. This encoding approach is called BSFQ local encoding scheme, in DEHP methodology. An example of AND operation using this local encoding scheme is shown in Fig. 1(c). Note that the AND gate operates asynchronously.

Encoded set-reset pulses are transferred by using a dual-rail Josephson transmission line (JTL), directed toward BSFQ cells [Fig. 1(b)], where operations are performed. For a special cell such as a BSFQ gate, these set-reset pulses are converted into superconducting flux levels, for performing Boolean operation according to its preset threshold of flux level [2]. The results 
(a)

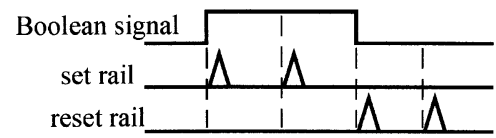

(b)

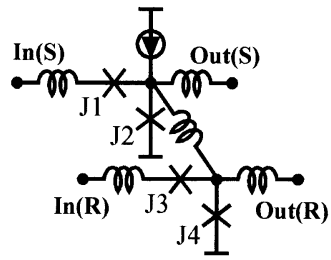

Fig. 2. (a) BSFQ global encoding scheme. (b) An error canceller cell represents an example of a BSFQ elementary cell. No converter is required for interchange of encoding, since there are escape junctions in all BSFQ elementary cells. Note that BSFQ error canceller is similar to RSFQ RS flip-flop [5], except the former one has an $\operatorname{Out}(\mathrm{R})$ rail.

are outputted in the form of set-reset pulses again for propagating to other BSFQ cells. For interfacing with external voltage level environment, BSFQ level-to-pulse converter and BSFQ pulse-to-level converter are used.

\section{System-LeVEL TIMING SCHEME}

In BSFQ local encoding scheme, process timing is obtained when there is a change in Boolean state encoded in data signals. However, if the state remains high or low, the process timing is unknown. Therefore, despite BSFQ combinational circuits operate asynchronously, BSFQ sequential circuits require global clock signal for synchronization. In order to make these sequential circuits operate asynchronously, we consider adding a new encoding scheme, BSFQ global encoding scheme [Fig. 2(a)], for channels requiring timing information that the local encoding scheme cannot provide. This global encoding scheme is similar to that of dual-rail logic [3], [4], where a data signal should be sent in either a set rail or a reset rail at every event of operation.

No converter is required for interchanging between global encoding and local encoding, thus facilitates hardware required. Consider a series of pulses, in the order shown in Fig. 2(a), arrives at the input of a BSFQ error canceller shown in Fig. 2(b). When two consecutive set pulses arrive, the latter pulse will be thrown off by escape junction $J 1$, maintaining the state "one" in the cell. As the same, when two consecutive reset pulses arrive, the cell will only output the first pulse and throw away the latter pulse through escape junction $J 3$. Hence, after processed by error canceller cell, the output sequence of the pulses will be just like Fig. 1(a). Since such escape junctions are with all BSFQ circuits, no additional converter is required for the interchange of encoding.

\section{HIERARCHICAL PIPELINING}

In BSFQ logic as well as CMOS logic, the simplest singlestage pipeline consists of a combinational logic block and a register. However, in conventional SFQ logic, such as RSFQ logic [5], the simplest pipeline limits to an elementary cell, since the elementary cell requires local clock to obtain processed result. Thus, BSFQ logic has an advantage, where one can build a big combinational logic block in the pipeline, as long as no feedback required in the block. This enables BSFQ combinational circuits to be simple in design and lower latency in operation, since local clock distribution, as well as time margins for a local

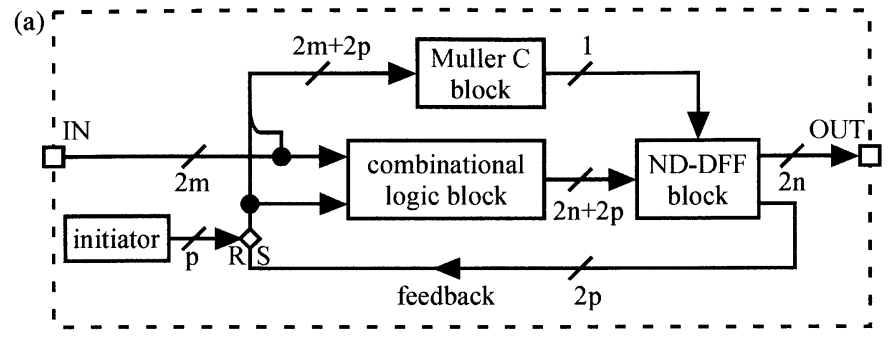

(b)

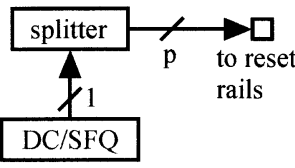

(c)

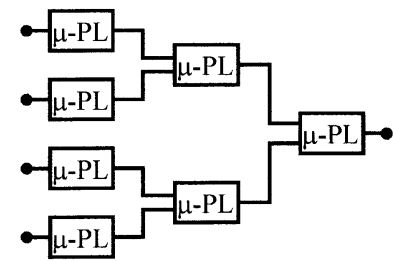

Fig. 3. (a) DEHP micro-pipeline. Variable $m, n, p$ shows bus width of the related channel. (b) Initiator is created by using a RSFQ DC/SFQ converter and a splitter block. Output rails of the initiator are connected to reset rails of the feedback channel. (c) An example of micro-pipeline (m-PL) network.

clock event, is not required at all. In order to enable BSFQ sequential circuits to have this advantage as well, we have developed hierarchical pipelining technique, for capsulating BSFQ sequential circuits into asynchronous functional block, so that these blocks can compose combinational functional block.

\section{A. Micro-Pipeline: Structure}

A DEHP micro-pipeline is the simplest sequential module, which has a feedback channel with an initiator, a combinational logic block, a BSFQ nondestructive D flip-flop (ND-DFF) block, and a Muller C-element block [Fig. 3(a)]. The ND-DFF block is similar to a CMOS register, which temporarily stores processed results. The Muller C-element block is a completion-waiting functional block, which outputs a ready signal if and only if all input signals are received.

BSFQ local encoding scheme is only used in communication inside combinational logic block, and communication between combinational logic block and ND-DFF block. The other parts of micro-pipeline, including the inputs and outputs, are using BSFQ global encoding scheme in communication.

\section{B. Micro-Pipeline: Operation}

1) Initialization: At system initialization stage, initiator in each micro-pipeline produces a SFQ pulse for each reset rail in the feedback channel. These pulses reset the related input rails in combinational logic block, and present as ready signals to Muller C-element block. The initiator consists of a RSFQ DC/SFQ cells and a splitter block [Fig. 3(b)]. It is activated by raising the input current of the DC/SFQ cells, which is controlled by either an external current source or an on-chip current source. The design is simple, since the input current path is independent of inductance, resulting in simple access to the required location.

2) Normal Routine: When input signals arrive at the micropipeline, they will be processed asynchronously by combinational logic block, and the results will be stored in ND-DFF block. On the other hand, the input signals also branch out to Muller C-element block, where their arrival is acknowledged. If 


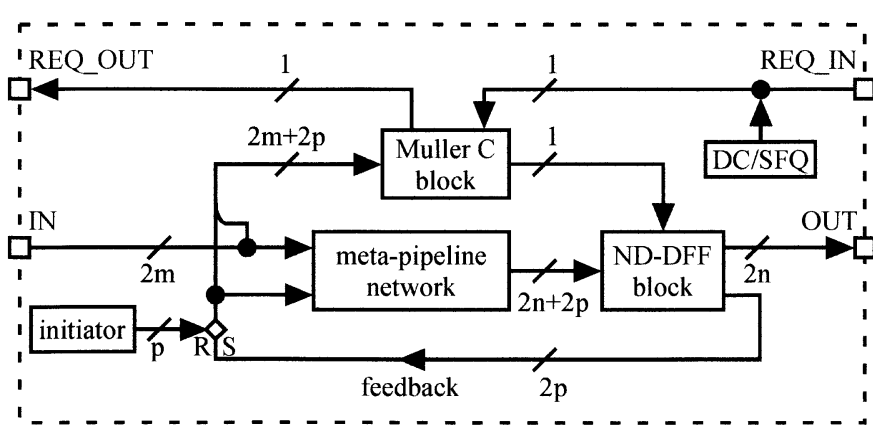

Fig. 4. DEHP pipeline. Initiator and DC/SFQ converter are used only in initialization of the pipeline. The diamond-shaped node indicates that all outputs of initiator connected to reset rails $(\mathrm{R})$ of feedback channel.

all input rails of Muller C-element block receiving signals, an output signal will be released to ND-DFF block, from where the stored results will be outputted to either the next micro-pipeline or the feedback channel. The whole process is repeated after the feedback signals return to the inputs of combinational logic block.

\section{Micro-Pipeline: Requirement}

1) Muller C-Element Block: In layout design, the shortest time interval for input pulses propagating from IN port to ND-DFF block through Muller C-element block, is adjusted by using several stages of JTL's. The adjusted time interval should be equal to a total of time interval for signals traveling from IN port to ND-DFF block through combinational logic block, and time margin for process variation. This can be easily calculated from logic simulation. If $T$ is the latency of combinational logic block, $\tau$ is the latency of feedback channel, $u$ is the latency of other auxiliary circuits, and $m$ is time margin for process variation, the total latency of one operating cycle for a micro-pipeline is $T+\tau+u+m$.

2) Feedback Channel: We may use low latency passive transmission lines instead of active JTL's for designing the feedback channel, so that the penalty of waiting feedback signals is lessened.

\section{Meta-Pipeline}

Substituting the combinational logic block in Fig. 3(a) with a network of micro-pipelines, we obtain a DEHP 1st-level meta-pipeline. No feedback is allowed in the micro-pipeline network, which is similar to that for combinational logic block. An example of micro-pipeline network is illustrated in Fig. 3(c). As the same, substituting the micro-pipeline network with a network of 1st-level meta-pipeline, we obtain DEHP 2nd-level meta-pipeline. By using this hierarchical pipelining method, we can construct an arbitrary FIFO sequential circuit.

\section{E. Pipeline}

A DEHP pipeline is the highest-level meta-pipeline, where backward communication of vacancy status or data request, can no longer be omitted. Fig. 4 illustrates structure of a pipeline, which is similar to that in Fig. 3(a), but has a request channel connecting to the adjacent pipelines, and has a DC/SFQ converter for initializing the request channel, directed from the suc-

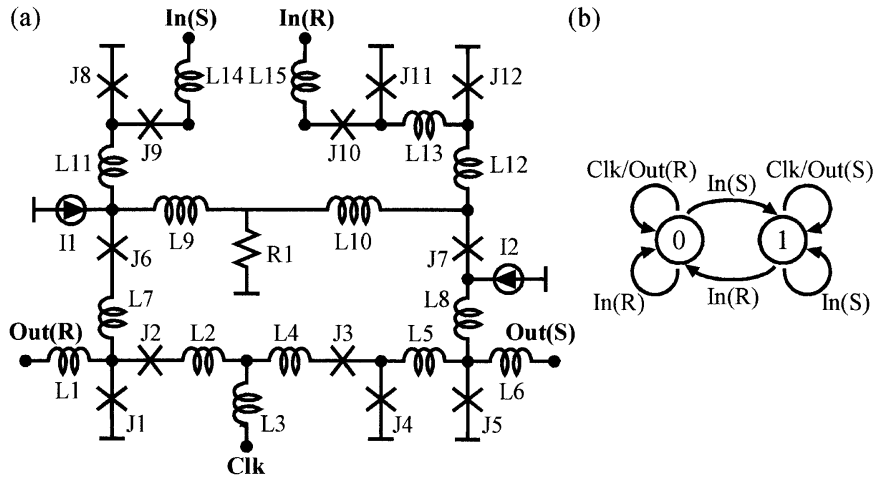

Fig. 5. (a) BSFQ nondestructive D flip-flop (D-DFF) cell. Nominal value: $I 1=0.23 \mathrm{~mA}, I 2=0.12 \mathrm{~mA}, J 1=J 8=0.14 \mathrm{~mA}, J 2=0.23 \mathrm{~mA}$, $J 3=J 9=0.20 \mathrm{~mA}, J 4=J 5=J 7=0.13 \mathrm{~mA}, J 6=0.12 \mathrm{~mA}$, $J 10=0.26 \mathrm{~mA}, J 11=J 12=0.17 \mathrm{~mA}, L 1=9.7 \mathrm{pH}, L 2=1.2$ $\mathrm{pH}, L 3=0.2 \mathrm{pH}, L 4=1.1 \mathrm{pH}, L 5=L 13=0.4 \mathrm{pH}, L 6=7.9$ $\mathrm{pH}, L 7=0.6 \mathrm{pH}, L 8=1.0 \mathrm{pH}, L 9=L 10=1.6 \mathrm{pH}, L 11=5.3$ $\mathrm{pH}, L 12=1.9 \mathrm{pH}, R 1=3.7 \Omega$. Parasitic inductances are omitted here but included in optimization. (b) Moore diagram of a ND-DFF cell.

ceeding pipeline. An example of a pipeline is a multiplier, an address calculator, an instruction decoder, etc. Thus, a DEHP pipeline is just like a single-stream FIFO executor of a specific job, and the backward request communication insures synchronization of the pipeline with adjacent pipelines, at the moment of reloading data. A demonstration of designing and high-speed testing of a DEHP pipeline has been carried out. The results will be presented in other paper soon.

\section{KEY CIRCUITS FOR DEHP METHODOLOGY}

\section{A. ND-DFF Circuit}

A BSFQ nondestructive D flip-flop (ND-DFF) is a nonvolatile memory cell, which stores a SFQ fluxon as its internal state, and the internal state is unchanged after a read-out process being carried out. In the read-out process, a timing signal is sent to ND-DFF, producing a set pulse or a reset pulse according to its internal state.

Schematic of a ND-DFF is shown in Fig. 5(a). ND-DFF is constructed by using the idea of RSFQ B flip-flop template [6]. This cell has three applications. First, it can be used as a data cache in a DEHP pipeline. Second, it can be used as a data encoder for the BSFQ global encoding scheme, where when switched by timing signals, a series of set or reset pulses is produced in the outputs, according to inputted Boolean signal in $\operatorname{In}(S)$ and $\operatorname{In}(R)$ rails. Third, it can be used as a direction switcher for single data rail, where inputted signals in Clk rail will be directed to either $\operatorname{Out}(\mathrm{S})$ rail or $\operatorname{Out}(\mathrm{R})$ rail, controlled by the inputted Boolean signal.

Parameter margins of ND-DFF are sensitive to parasitic inductance, where a bigger parasitic inductance leads to a narrower margin. Thus, after incipient design of the cell, the parasitic inductances are extracted, and the whole cell is re-optimized. Optimized BSFQ ND-DFF, including consideration of parasitic inductance, has simulated global bias margin ranging from $-29 \%$ to $+26 \%$ at $10 \mathrm{GHz}$. In simulation, the critical current density, IcRn product and McCumber parameter of a shunted Josephson junction are assumed $2.5 \mathrm{kA} / \mathrm{cm}^{2}, 0.37 \mathrm{mV}$, 
(a)

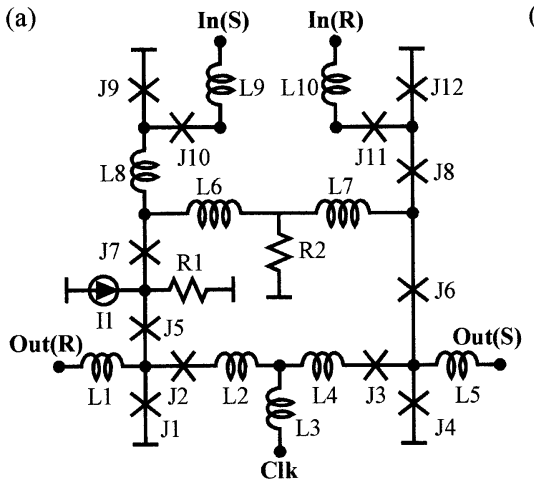

(b)

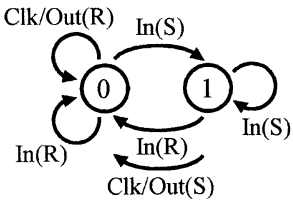

Fig. 6. (a) BSFQ destructive $\mathrm{D}$ flip-flop (D-DFF) cell. Nominal value: $I 1=$ $0.20 \mathrm{~mA}, J 1=J 5=J 9=0.16 \mathrm{~mA}, J 2=J 4=0.21 \mathrm{~mA}, J 3=0.24$ $\mathrm{mA}, J 6=J 7=0.27 \mathrm{~mA}, J 8=0.14 \mathrm{~mA}, J 10=0.29 \mathrm{~mA}, J 11=0.13$ $\mathrm{mA}, J 12=0.12 \mathrm{~mA}, L 1=9.7 \mathrm{pH}, L 2=1.2 \mathrm{pH}, L 3=0.2 \mathrm{pH}, L 4=1.1$ $\mathrm{pH}, L 5=7.9 \mathrm{pH}, L 6=L 7=1.3 \mathrm{pH}, L 8=2.6 \mathrm{pH}, L 9=L 10=1.5 \mathrm{pH}$, $R 1=R 2=3.7 \Omega$. Parasitic inductances are omitted here but included in the optimization. (b) Moore diagram of a D-DFF cell.

and unity, respectively, where $I c$ is critical current, and $R n$ is normal resistance of a shunted Josephson junction.

A Moore diagram for ND-DFF is shown in Fig. 5(b). For understanding operating mechanism of ND-DFF, first we consider the internal state of this cell is "zero." When a signal arrives at $\mathrm{In}(\mathrm{S})$ rail, it switches junction $J 8$ and $J 6$, and the internal state of the cell becomes "one." Successive set pulses will be thrown away by escape junction $J 9$, for maintaining the state "one" in the cell. Then, if a reset pulse arrives at $\operatorname{In}(\mathrm{R})$, it will switch junction $J 11, J 12$, and $J 7$, and restore the internal state of the cell. Successive reset pulses will be thrown away by escape junction $J 10$. On the other hand, if a timing signal arrives at Clk rail when the cell is in state "zero," it will switch junction $J 3, J 1$, and $J 6$, and produce a reset pulse at the $\operatorname{Out}(\mathrm{R})$ rail. Switching of junction J6 maintains the state "zero" of the cell. On the contrary, if a timing pulse arrives at Clk rail when the cell is in state "one," it will switch junction $J 2, J 4, J 5$, and $J 7$, consecutively, and produce a set pulse to the Out(S) rail. Switching of junction $J 7$ maintains the state "one" for the cell, ensuring nondestructive read-out of stored information.

\section{B. D-DFF Circuit}

A BSFQ destructive D flip-flop (D-DFF) is a volatile memory cell that the internal state restores to its initial state "zero" when switched by a timing signal. This cell is mainly used to build up a BSFQ shift-register. Optimized D-DFF, which includes consideration of parasitic inductance in layout, has simulated bias margins ranging from $-30 \%$ to $+32 \%$ at $10 \mathrm{GHz}$. Schematic of

a D-DFF is shown in Fig. 6(a), and its Moore diagram is shown in Fig. 6(b).

Consider the cell is at state "zero." When a signal arrives at $\operatorname{In}(\mathrm{S})$ rail, it switches junction $J 9$ and $J 5$, resulting in the cell turning into state "one." Then, if a reset pulse arrives at $\operatorname{In}(\mathrm{R})$, it will switch junction $J 12$ and $J 6$, and restore the internal state of the cell to state "zero" again. On the other hand, if a timing signal arrives at Clk rail when the cell is in state "zero," it will switch junction $J 3, J 1$, and $J 7$, and produce a reset pulse at the Out( $\mathrm{R})$ rail. On the contrary, if a timing pulse arrives at Clk rail when the cell is in state "one," it will switch junction $J 2, J 4$, and $J 8$, consecutively, and produce a set pulse to the Out(S) rail. The internal state of D-DFF restores to state "zero" after the switching of junction $J 8$.

\section{CONCLUSION}

We have presented a novel global self-timing methodology, DEHP, for constructing a globally asynchronous locally asynchronous BSFQ system. In this methodology, two encoding schemes are used for representing a Boolean signal, where one of them provides sufficient timing information for BSFQ local cell, and the other one provides sufficient information for global functional block. Besides, the methodology is using hierarchical pipelining, where the self-timed micro-pipeline, meta-pipeline, and pipeline are used to build an arbitrary asynchronous BSFQ system. A nonvolatile memory cell named ND-DFF and a volatile memory cell named D-DFF are constructed to achieve this goal.

\section{REFERENCES}

[1] H. Kodaka, T. Hosoki, and Y. Okabe, "Single flux quantum level circuit using new DC/SFQ," IEEE Trans. Appl. Supercond., vol. 9, pp. 3729-3732, Jun. 1999.

[2] C. K. Teh and Y. Okabe, "New BSFQ circuit designs with wide margins," IEEE Trans. Appl. Supercond., vol. 11, no. 1, pp. 970-973, Mar. 2001 .

[3] Z. J. Deng, S. R. Whiteley, and T. Van Duzer, "Data-driven self-timing of RSFQ digital integrated circuits," in Ext. Abst. of 5th Int. Supercond. Electron. Conf., 1995, pp. 189-191.

[4] M. Maezawa, I. Kurosawa, M. Aoyagi, H. Nakagawa, Y. Kameda, and T. Nanya, "Pulse-driven dual-rail logic gate family based on rapid singleflux-quantum (RSFQ) devices for asynchronous circuits," in Proc. of 2nd Int. Symp. on Adv. Res. in Asynchronous Circ. and Syst., 1996, pp. 134-142.

[5] K. K. Likharev and V. K. Semenov, "RSFQ logic/memory family: A new Josephson-junction digital technology for sub-terahertz-clock-frequency digital systems," IEEE Trans. Appl. Supercond., vol. 1, pp. 3-28, Mar. 1991.

[6] S. V. Polonsky, V. K. Semenov, and A. F. Kirichenko, "Single flux quantum B flip-flop and its possible applications," IEEE Trans. Appl. Supercond., vol. 4, pp. 9-18, Mar. 1994. 